\title{
Verknüpfen und Urteilen*
}

\author{
Ein Experiment zur Wirkung medialer Value-Frames
}

\section{Bertram Scheufele}

Der Beitrag untersucht die Effekte medialer Value-Frames am Beispiel der Diskussion über den potenziellen EU-Beitritt der Türkei. Ein zweifaktorielles Experiment unterschied drei politische Werte bzw. Ziele ("Internationale Sicherbeit“, „Menschenrechte“ und „Wirtschaftliche Stabilität") und zwei Politiker (Merkel, Steinmeier). Erstens wird geprüft, ob die Rabmung eines Politikers als „, Garant " für einen dieser Werte dazu fübrt, dass Rezipienten den Politiker kognitiv stärker mit diesem Wert verknüpfen als bei anderem Value-Framing (Verknüpfungseffekt). Zweitens wird untersucht, wie sich das auf Urteile über den Politiker auswirkt (Priming-Effekt). Den Befunden zufolge ist der Verknüpfungseffekt stärker für Werte mit größerer Bedeutung für die meisten Bürger eines Landes. Zudem gibt es gewisse Hinweise auf Priming-Effekte etwa im Hinblick auf die Direktwablabsicht der Probanden. Diese werden aber vom aktuellen Meinungsklima im Bundestagswablkampf 2009 gebrochen. Die politische Grundhaltung der Probanden verstärkte die Effekte, die moderierende Rolle der subjektiven Werte-Relevanz ist dagegen nicht eindeutig.

Schlüsselwörter: Framing, Priming, Werte, Medienwirkung, Politische Kommunikation, Wahlen

\section{Framing-Effekte}

Klassifiziert man Framing-Effekte nach Typen von Medien-Frames (vgl. z. B. Nelson/ Willey 2001: 246-248; B. Scheufele 2004: 412-415), dann lassen sich z. B. Studien zur Wirkung von „episodic frames“ (Iyengar 1991), „issue frames“ (Nelson/Oxley 1999), „strategic frames“ (Cappella/Jamieson 1997) oder zu Effekten von „value-frames“ (Shah et al. 1996) unterscheiden. Der vorliegende Beitrag setzt sich mit Value-Framing-Effekten auseinander. Ganz allgemein ist ein Frame ein Bezugsrahmen oder eine Perspektive, von der aus Themen, Ereignisse und Akteure betrachtet werden (vgl. z. B. Entman 1993: 52; Reese 2001: 11). Framing „refers to modes of presentation that journalists and other communicators use to present information in a way that resonates with existing underlying schemas among their audience“ (D. Scheufele/Tewksbury 2007: 12). Auf diese Weise aktivieren Medien nicht nur bestimmte Kognitionen bei Rezipienten, sondern können auch deren Urteile oder Entscheidungen prägen (vgl. z. B. Price/Tewksbury 1997; B. Scheufele 2003, 2004; Matthes 2007).

Framing-Effekte haben Schnittstellen zu Priming-Effekten (vgl. dazu Peter 2002). Der überzeugendste Vorschlag zur Integration beider Ansätze stammt von Price/Tewksbury (1997): Indem Medien ein Thema auf eine bestimmte Weise rahmen, signalisieren sie Rezipienten, welche ihrer Schemata bzw. Wissenseinheiten sich auf das Thema anwenden lassen. Das ist der Anwendbarkeits- oder Framing-Effekt. Wenn ein Zeitungsartikel z. B. einen Krieg in den Rahmen von Menschenrechten stellt, dürften Rezipienten diesen Krieg eher als Menschenrechtsfrage denn als Frage internationaler Sicherheit ansehen. Die auf diese Weise aktivierten Schemata rund um Menschenrechte behalten aber

* Der Autor dankt den anonymen Gutachtern für ihre hilfreichen Anmerkungen. 
für einige Zeit ihr Aktivationsniveau. Sie sind daher für spätere Urteile leichter zugänglich als andere Schemata. Das bezeichnen die Autoren als Zugänglichkeits- bzw. Priming-Effekt. Damit fungiert ein Medien-Frame also gleichsam als Prime für spätere Urteile z. B. über einen Politiker.

Allerdings konkurrieren die mittels Framing aktivierten Schemata immer auch mit Schemata, die den Rezipienten ebenfalls leichter zugänglich sind - etwa weil sie zuvor wiederholt aktiviert worden sind. Wie beide zusammenspielen, lässt sich mit dem Konzept mentaler Modelle (z. B. Johnson-Laird 1980) erklären (vgl. B. Scheufele/D. Scheufele 2009): Rezipienten haben z. B. schon Vorstellungen von Kriegen, die zunächst das mentale Modell prägen, das sich Rezipienten von jenem neuen Krieg bilden, über den sie ein Zeitungsartikel informiert $\left(t_{1}\right)$. Rahmt der Artikel den Krieg als Menschenrechtsfrage, werden alle Schemata der Rezipienten rund um Menschenrechte anwendbar und aktiviert. Damit hat sich das Bild der Rezipienten von dem Krieg bereits verändert $\left(\mathrm{t}_{2}\right)$, wird aber noch stark vom Vorwissen geprägt. Rahmen viele Medien den Krieg immer wieder auf diese Weise, werden sich die kognitiven Koordinaten der Rezipienten jedoch sukzessive verändern $\left(t_{3}\right)$, so dass sie möglicherweise $\mathrm{zu}$ anderen Schlussfolgerungen als zuvor kommen.

Mit vergleichbaren Überlegungen ergänzt B. Scheufele (2004: 409-410) das Modell von Price/Tewksbury (1997) um das Argument, wiederholtes Framing könne die Schemata von Rezipienten neu „verlinken “: Wer die Bundeswehr bislang mit Landesverteidigung verknüpfte, wird nun an Menschenrechtsverletzungen und humanitäre Bundeswehreinsätze denken (vgl. B. Scheufele/Gasteiger 2007). Auch Brewer et al. (2003: 496) nehmen an, dass „[e]xposure to the frame could also create an association where none previously existed“. Damit kann Framing entweder die im kognitiven Netzwerk bereits bestehenden Assoziationen aktivieren oder sie erst etablieren (vgl. auch Price/Tewksbury 1997: 188). Eine empirische Überprüfung dieser Argumente steht aber noch aus (vgl. Matthes 2007: 112). Dafür wäre auch ein Design mit sehr vielen Messzeitpunkten notwendig. Ein Anfang wäre zumindest gemacht, wenn man die Verknüpfung von Wissenseinheiten tatsächlich messen würde. In Bezug auf kognitive Framing-Effekte wird aber meist nur geprüft, ob die Gedankenprotokolle der Probanden mit den induzierten Frames korrespondieren (z. B. DeVreese/Boomgaarden 2003). Eine Ausnahme bilden aber Brewer et al. (2003). Sie verglichen die Meinung über Mexiko und Kolumbien u. a. bei Lesern eines Artikels, der über das Drogenproblem in den USA berichtete, und Lesern eines Artikels, der die Länder als Drogenproduzenten präsentierte. Dass die Probanden eine Assoziation zwischen Frame und Ländern erst aufbauten, konnte zwar aus dem Gruppenvergleich, damit aber nur indirekt erschlossen werden.

\section{Modell der Value-Framing-Effekte}

\subsection{Verknüpfungs-und Priming-Effekte}

Ein zentrales Defizit der Forschung zu Framing-Effekten besteht darin, dass oft recht spezifische, themenabhängige Frames betrachtet werden. Die wenigen Versuche, themenübergreifende Frames (z. B. conflict, economy) zu definieren (vgl. z. B. Neuman et al. 1992; Semetko/Valkenburg 2000), überzeugen nur bedingt. Denn diese Frames sind eher Nachrichtenfaktoren bzw. Themen (vgl. z. B. B. Scheufele 2004: 419) und theoretisch wenig fundiert. Die bislang überzeugendste Konzeption für themenübergreifende Rahmen sind Value-Frames, also die Konzeption allgemeiner Werte, genereller Grundsätze bzw. Ziele als Bezugsrahmen. 
Bisherige Studien fragten nach der Wirkung von Value-Frames wie „Sonderrechte vs. Gleichberechtigung “ (Price et al. 2005) und „Gleichheit vs. Moral“ (Brewer 2002) beim Thema gleichgeschlechtlicher Ehe oder von Frames wie „Individualismus vs. Humanität“ (Shen/Edwards 2005) und „Ethik vs. Pragmatik“ (Shah et al. 1996; Domke et al. 1998) beim Thema staatlicher Wohlfahrt sowie „Freie Rede vs. Öffentliche Ordnung“ beim Thema Ku-Klux-Klan (Nelson et al. 1997). Dieser zweifellos nicht vollständige Überblick zeigt, dass manche Studien grundlegende gesellschaftliche Werte betrachten, andere Studien politikfeldspezifische Grundsätze und wieder andere sozialpsychologisch zu begründende Werte fokussieren. Damit ist festzuhalten, dass das Konstrukt Value-Frame recht breit konzipiert wird und auf übergeordnete Rahmen im Sinne von Werten, Zielen oder Grundsätzen verweist.

Schwartz/Bardi (2001: 269) definieren Werte als „desirable, transsituational goals, varying in importance, that serve as guiding principles in peoples's lives“ - oder einfacher als Vorstellungen des Wünschenswerten für Individuen oder die Gesellschaft (vgl. Kluckhohn 1951: 395). Nach Gabriel (2009: 31) sind politische Werte bzw. Wertorientierungen die „von den Mitgliedern einer politischen Gemeinschaft als gemeinsam anerkannten Vorstellungen von den anzustrebenden Zielen des politischen Zusammenlebens und den zur Erreichung dieser Ziele angemessenen Mittel“. Bei der Frage nach (politischen) Zielen geht es also letztlich immer um die Frage nach der Verwirklichung von (politischen) Werten.

Der vorliegende Beitrag fragt nach den Wirkungen einer medialen Rahmung mittels solcher politischen Werte bzw. Ziele. Bisherige Studien zu Value-Framing-Effekten berücksichtigten ähnliche abhängige Variablen wie Studien zur Wirkung anderer Frames. Brewer (2002), Brewer/Gross (2005) und Shen/Edwards (2005) untersuchten, ob Gedankenprotokolle der Rezipienten die experimentell manipulierten Value-Frames widerspiegelten. Price et al. (2005: 185) weisen jedoch darauf hin, dass es keine simple Übernahme von Frames geben muss, denn ,alternative frames will nevertheless be invoked“. Auch Brewer (2002: 311-312) zeigte, dass ein konkurrierender Frame die Wirkung des Kernframes abschwächen kann. Wie Medien-Frames mit dem Vorwissen der Rezipienten zusammenspielen, wurde mit dem Konzept mentaler Modelle beschrieben. Slothuus (2008) fragte wiederum nach Kausalattributionen und somit nach weitergehenden Elaborationen. Die meisten Studien zielen auf Meinungen z. B. zu Wohlfahrtzuwendungen (Slothuus 2008) oder zur Homosexuellenbewegung (Price et al. 2005). Nur wenige Studien untersuchten die Effekte auf das Entscheidungsverhalten (z. B. Shah et al. 1996). Domke et al. (1998) fanden Hinweise auf weitergehende Effekte. Sie prüften, ob die ethische Rahmung einer Gesundheitsreform dazu führt, dass Probanden die im Artikel genannten Kandidaten eher anhand ihrer Integrität beurteilen - das Gegenteil war jedoch der Fall. Allerdings strahlte die ethische Rahmung der Gesundheitsreform auch auf die Interpretation, entfernterer' Themen (z. B. Bildung) aus. Gleichwohl bemängeln Domke et al. (1998: 70), dass noch zu wenig bekannt sei über „the role of values for individuals, particularly the linkages between values and political attitudes“.

Das vorliegende Experiment greift diesen Hinweis auf und untersucht zwei Typen von Value-Framing-Effekten:

(1) Wenn Medien einen Politiker in den Rahmen eines politischen Wertes oder Ziels stellen, dann dürften sie ihn/sie zugleich als förderlich oder hinderlich für diesen Wert oder dieses Ziel darstellen. So erscheint Angela Merkel als „Garant“ für Menschenrechte, wenn ein Zeitungsartikel sie damit zitiert, dass sie den EU-Beitritt der Türkei wegen Menschenrechtsverletzungen ablehne. Dieses Value-Framing dürfte dazu führen, dass Rezipienten die Kanzlerin zumindest kurzfristig stärker mit diesem Wert verbinden als 
bei anderer Rahmung. Das kann man als Verknüpfungseffekt bezeichnen. Mit Brewer et al. (2003: 496) lässt er sich einerseits damit erklären, dass solche Assoziationen in den Köpfen der Rezipienten bereits bestehen und durch das Value-Framing des Artikels nur aktiviert werden. Value-Framing kann Verknüpfungen in den Köpfen der Rezipienten aber auch erst etablieren. Solche Verknüpfungen werden sich bei konsonanter und kumulativer Rahmung dann auch kognitiv einprägen.

(2) Folgt man Price/Tewksbury (1997), dann bleibt die durch Value-Framing aktivierte Assoziation zwischen Politiker und Wert eine Zeit lang beim Rezipienten kognitiv präsent und kann daher als Urteilsgrundlage für ein späteres Urteil über den Politiker dienen. Das wäre dann ein Priming-Effekt. Wenn ein Medienbeitrag einen Politiker als „Garant“ für einen politischen Wert bzw. ein politisches Ziel präsentiert, dürften Rezipienten den Politiker also nicht nur mit diesem Wert verknüpfen, sondern ihn auch positiver beurteilen als ohne solches Value-Framing. Wird dagegen z. B. Angela Merkel in einem Artikel als „Gefahr“ für Gerechtigkeit dargestellt, dann werden Rezipienten die Kanzlerin negativer bewerten als ohne solche Rahmung. Das lässt sich auch mit Rosenbergs (1956) Theorie affektiv-kognitiver Konsistenz erklären, die schon von der Wirkungsperspektive der Theorie instrumenteller Aktualisierung (vgl. Kepplinger et al. 1991) aufgegriffen wurde: Nach Rosenberg entwickelt ein Mensch eine positive Einstellung gegenüber einem Objekt (z. B. Merkel), wenn dieses instrumentell nützlich (z. B. „Garant“) für einen zentralen Wert (z. B. internationale Sicherheit) ist.

\subsection{Moderierende Variablen}

Beide Effekte dürften durch verschiedene Variablen moderiert werden. Eine Rolle spielt erstens die Bedeutung im politisch-gesellschaftlichen Wertekanon, also die Frage, ob ein Wert bzw. Ziel zentrale Bedeutung für die meisten Bürger eines Landes hat. Zu den „grundlegenden Politikziele[n der Bundesrepublik gehören vor allem ...] äußere und innere Sicherheit, Freiheit, Wohlstand, sozioökonomische Sicherheit und Gleichheit sowie Umweltschutz“ (Fuchs/Rohrschneider 2005: 340). Über diese Ziele, die auf entsprechende Werte verweisen, bestehe „weitgehender Konsens in der Bevölkerung“ (ebd.: 341). Strittig sei aber, welcher Politiker bzw. welche Partei kompetenter in der Erreichung des betreffenden Ziels sei bzw. wer - in der Begrifflichkeit dieses Beitrags - für den jeweiligen Wert ein „Garant“ oder eine „Gefahr“ ist. Nach Korte/Fröhlich (2004: $115)$ ist die „Sehnsucht nach Sicherheit [...] ein prägnantes Kennzeichen der politischen Kultur der Deutschen“. Eine vergleichbare Bedeutung von Sicherheitsfragen fanden Schwartz/Bardi (2001: 275) in ihrer Wertestudie in mehreren Ländern. Auch in einer GMS-Umfrage in Deutschland rangierte Sicherheit weit oben (vgl. Jung 2005).

Bei einem solch zentralen Wert bzw. Ziel sollten die postulierten Effekte stärker sein als bei Werten, die geringere Bedeutung für die meisten Menschen haben. Als Beispiel denke man sich einen Artikel über den EU-Beitritt der Türkei und die Haltung Angela Merkels (vgl. Appendix B). Wenn die Kanzlerin im Artikel deutliche Kritik an der sicherheitspolitischen Lage in der Türkei äußert, erscheint sie als „Garant“ für internationale Sicherbeit. Bei dieser Rahmung dürften die beschriebenen Effekte stärker sein als bei einem Artikel, der den Rahmen wirtschaftlicher Stabilität in der Türkei anlegt. Wirtschaftliche Stabilität ist ein wichtiges Ziel der Wirtschaftspolitik auch der Bundesrepublik (vgl. Kevenhörster 2006: 101), hat aber geringere Bedeutung für den deutschen Normalbürger als internationale Sicherheit. Denn die Gefahr, z. B. Opfer von Terror- 
akten zu werden, ${ }^{1}$ ist greifbarer als die für ihn zunächst eher vage Instabilität des Wirtschaftssystems der Türkei. Die Frage von Menschenrechten in der Türkei betrifft wiederum vor allem die dortige Bevölkerung. Das dürfte für den deutschen Durchschnittsbürger ebenfalls geringere Bedeutung haben als die Frage internationaler Sicherheit, die immer auch das eigene Land betrifft - nicht erst seit dem 11. September 2001.

Moderierend dürfte zweitens die individuelle Werterelevanz des Rezipienten wirken. Wenn Medien z. B. Merkel als „Garant“ für Sicherheit darstellen, werden Rezipienten sie noch stärker mit diesem Wert verknüpfen, wenn Sicherheit für sie persönlich wichtig ist. Dafür sprechen Befunde bei Shen/Edwards (2005: 802-803). Das lässt sich mit kognitionspsychologischen Netzwerktheorien (vgl. als Überblick z. B. Operario/Fiske 2004: 127-129) erklären: Das kognitive System eines Menschen dürfte aufnahmefähiger für einen Value-Frame sein, wenn es schon Strukturen rund um diesen Wert ausgebildet hat - und das ist bei hoher persönlicher Relevanz wahrscheinlich. Andererseits zeigen schematheoretische Befunde (z. B. Fiske et al. 1983) aber auch, dass elaborierte Kognitionen recht robust gegenüber neuen Informationen sind. Daher ist durchaus auch denkbar, dass der Value-Frame eine Assoziation dann etabliert, wenn Rezipienten dem betreffenden Wert geringe Relevanz zuschreiben und noch kaum Kognitionen rund um den Wert entwickelt haben. Empirische Belege in diese Richtung bietet z. B. Sloothuus (2008: 18).

Bei Urteilen über Politiker wird drittens die politische Grundhaltung von Bedeutung sein. Mit Price et al. (2005: 202) kann man von „ideological resonance“ sprechen. Wer eine Partei oder deren Kandidaten gut findet, ist anfälliger für das Value-Framing dieser Partei oder dieses Kandidaten. So konnten Shah et al. (1996: 325-326) zeigen, dass die Wirkung eines Ethik-Frames bei christlichen Probanden noch stärker ausfiel.

\section{Hypothesen und Forschungsfragen}

Der erste zu untersuchende Value-Framing-Effekt ist der Verknüpfungseffekt: Die Rahmung eines Politikers mittels eines politischen Wertes bzw. Ziels dürfte dazu führen, dass Rezipienten den so gerahmten Politiker mit diesem Wert bzw. Ziel kognitiv verknüpfen.

Hypothese H1: Leser eines Beitrags, der einen Politiker in den Rabmen eines Wertes stellt, verbinden den Politiker stärker mit diesem Wert als Leser eines Artikels mit anderem Value-Frame.

Dabei werden aber auch die erwähnten moderierenden Faktoren wirksam. Erstens kann der Stellenwert im politisch-gesellschaftlichen Wertekanon, also die Bedeutung des Wertes für die meisten Bürger eine Rolle spielen. Der Verknüpfungseffekt dürfte bei Werten mit großer Bedeutung für die meisten Bürger (z. B. internationale Sicherheit) stärker ausfallen als bei anderen Werten (z. B. wirtschaftliche Stabilität, Menschenrechte in anderen Ländern).

Hypothese H2: Der Verknüpfungseffekt ist stärker bei Werten mit großer Bedeutung für die meisten Menschen als bei Werten mit geringer Bedeutung für die meisten Menschen.

Zweitens dürfte die Relevanz, die ein Mensch dem Wert bzw. Ziel subjektiv zuschreibt, eine Rolle spielen. Allerdings sind die bisherigen Befunde zu dieser Drittvariablen eher widersprüchlich. Daher wird folgende Forschungsfrage formuliert:

1 Diese wird im späteren Stimulusartikel auch explizit erwähnt (vgl. Appendix B). 
Forschungsfrage F1: Ist der Verknüpfungseffekt stärker bei Werten, denen Rezipienten individuell hohe Relevanz zuschreiben, oder stärker bei Werten, denen sie individuell geringe Relevanz zuschreiben?

Drittens dürfte die politische Grundhaltung moderieren. Wenn ein Medienbeitrag einen Politiker als „Garant“ für einen Wert präsentiert, dürften besonders jene Rezipienten, die dem Politiker in der politischen Grundhaltung nahestehen, ihn mit dem betreffenden Wert verknüpfen.

Hypothese H3: Für Politiker, deren Partei mit der politischen Grundhaltung des Rezipienten korrespondiert, ist der Verknüpfungseffekt stärker als für Politiker aus dem anderen politischen Lager.

Schließlich kann der Value-Frame als Prime für Urteile über den Politiker fungieren: Wenn ein Medienbeitrag einen Politiker als „Garant“ für einen Wert präsentiert, dürften Rezipienten den Politiker positiver beurteilen als ohne solches Value-Framing. Je stärker der Verknüpfungseffekt, desto deutlicher dürfte also auch der Priming-Effekt sein. H2 postulierte, dass der Verknüpfungseffekt für Werte mit großer Bedeutung für die meisten Menschen stärker ausfällt als für gesellschaftlich weniger bedeutsame Werte. Daraus ergibt sich folgende Hypothese:

Hypothese H4: Leser eines Beitrags, der einen Politiker als „Garant“ eines für die meisten Menschen bedeutsamen Wertes präsentiert, bewerten den Politiker positiver als Leser eines Artikels, der den Politiker als „Garant“ eines weniger bedeutsamen Wertes präsentiert.

Auch hier dürften die politische Grundhaltung und die subjektive Werte-Relevanz auf die bereits beschriebene Weise moderieren.

\section{Design und Methode}

Die Hypothesenprüfung erfolgte im Rahmen eines zweifaktoriellen Experiments. Die Probanden lasen einen Zeitungsartikel, in dem sich ein deutscher Politiker zu einem EUBeitritt der Türkei äußerte. Der EU-Beitritt war kein Thema im Bundestagswahlkampf 2009. Diese Tatsache machte es unwahrscheinlich, dass Probanden durch die aktuelle Berichterstattung schon vorab geprimt worden waren. Der Zeitungsartikel wurde auf Basis von Medienbeiträgen, wissenschaftlichen und anderen Quellen erstellt (vgl. Appendix A). Die Versionen unterschieden sich nur in Überschriften und Schlüsselwörtern (vgl. Appendix B).

(1) Der erste Faktor war der Value-Frame. Die eine Artikelversion stellte den Beitritt in den Rahmen von Menschenrechten, die zweite in den Rahmen internationaler Sicherheit und die dritte in den Rahmen wirtschaftlicher Stabilität. Diese Frames repräsentieren zentrale Argumente zum EU-Beitritt. ${ }^{2}$ Wie schon dargelegt, haben die drei Werte bzw. Ziele unterschiedlich starke Bedeutung für die meisten Deutschen: Internationale Sicherheit betrifft immer auch Deutschland, zumal Sicherheitsfragen in den genannten Wertestudien hoch rangieren. ${ }^{3}$ Menschenrechtsfragen in der Türkei betreffen zunächst nur die dortige Bevölkerung. Und wirtschaftliche Stabilität dürfte für den Normalbürger weniger greifbar sein als die Bedrohung der Sicherheit des eigenen Landes. Der Politiker argumentierte kritisch in Bezug auf den Wert (z. B. „Merkel: ,Menschenrechte müssen

2 Die Beschränkung auf drei Werte hatte forschungsökonomische Gründe.

3 Die Bedeutung internationaler Sicherheit zeigt sich im Übrigen auch in der gemeinsamen Außen-, Sicherheits- und Verteidigungspolitik der EU (vgl. www.swp-berlin.org/transfer/Lissa bon/pdf/gasp.pdf sowie www.swp-berlin.org/transfer/Lissabon/pdf/gsvp.pdf [19.02.2010] sowie in aktuellen Wahlprogrammen (vgl. CDU/CSU 2009: 84-92; SPD 2009: 68-72)). 
eingehalten werden’“). Damit erschien er durchweg als „Garant“ für diesen Wert, was unter anderem für die Überprüfung von $\mathrm{H} 4$ entscheidend war.

Als zweiter Faktor wurde der Politiker variiert. Im einen Fall war es Angela Merkel (CDU/CSU), im anderen Frank-Walter Steinmeier (SPD). Die Entscheidung für diesen Faktor resultierte aus zwei Überlegungen: Erstens sollten die postulierten Effekte zunächst einmal politikerunabhängig sein. Zweitens ist aber denkbar, dass die Effekte stärker sind, wenn das Thema in den Zuständigkeitsbereich eines Politikers fällt, da z. B. Steinmeier als damaligem Außenminister mehr Kompetenz in der Beurteilung der Beitrittsfrage zugeschrieben werden dürfte - auch wenn sich Merkel ebenfalls schon dazu geäußert hat. ${ }^{4}$ Drittens konnte mit diesem Faktor die moderierende Rolle der politischen Grundhaltung verlässlich geprüft werden.

Eine Kontrollgruppe ohne Artikel ergänzte die sechs Experimentalgruppen (vgl. Tabelle 1). Das Experiment wurde im Rahmen eines Seminars realisiert. Versuchsleiter waren die 28 Studierenden. ${ }^{5}$ Sie sollten jeweils acht Probanden kontaktierten und erhielten drei Altersgruppen (18-29 Jahre, 30-49 Jahre, ab 50 Jahre) und zwei Geschlechter als nichtrepräsentative Quotenvorgabe. Die Angaben der Studierenden zur Quotenerfüllung waren der Ausgangspunkt der Randomisierung. Zunächst wurden alle kontaktierten Probanden einer Quotengruppe gelistet. Dann wurden die sieben Experimentalbedingungen so lange über die Probanden dieser Quotengruppe rotiert, bis jeder Proband einer Bedingung zugewiesen war. Dazu ein Beispiel: In der Gruppe 18- bis 29-jähriger Männer waren 49 Personen vorkontaktiert worden. Der erste Befragte dieser Quotengruppe wurde der Experimentalbedingung 1 zugewiesen, der zweite der Bedingung 2 usw., der achte Befragte wieder der Bedingung 1, der neunte der Bedingung 2 usw. Analog wurde für die übrigen fünf Quotengruppen verfahren. ${ }^{6}$ Die Zielgröße waren 217 Probanden. Allerdings gab es Ausfälle: 20 Probanden hatten bei zwei oder mehr Fragen zu den abhängigen Variablen nur teilweise angekreuzt (z. B. für drei von neun Items) oder z. B. bei allen neun Items den niedrigsten oder durchweg den höchsten Wert angekreuzt. In diesen Fällen waren die Antworten offenkundig unbrauchbar. Fünf Probanden waren durch die Versuchsleiter der falschen Experimentalbedingung zugewiesen worden und zwei Probanden waren eingeplant, nahmen aber dann doch nicht teil. Beibehalten wurden 11 Probanden, deren Alter bzw. Geschlecht zwar nicht der Quote entsprach, bei denen aber die Experimentalbedingung stimmte. Denn dadurch verschob sich lediglich der Alters- bzw. Geschlechteranteil in den sieben Experimentalbedingungen (vgl. Tabelle 1). Da die Ausfälle trotz Zufallsrotation am stärksten die zweite Gruppe betrafen, erfolgte am Ende der Durchführungsphase (2.-20.7.2009) eine Nacherhebung für drei Probanden dieser Gruppe. Jeder dieser „neuen“ Probanden entsprach jeweils einem Ausfall in dieser Quotengruppe. ${ }^{7}$ Insgesamt gab es damit 193 Probanden. Die

4 Vgl. z. B. www.spiegel.de/politik/ausland/0,1518,441075,00.html [19.02.2010].

5 Ich danke allen Teilnehmer/innen für ihr Engagement. Exemplarisch hervorheben möchte ich Anika Dornieden, Sebastian Ernst, Katrin Hesse und Christin Kalupke. Zudem gilt mein Dank Manuela Bischoff für ihr hilfreiches Thesenpapier zur Debatte um den EU-Beitritt der Türkei (vgl. Appendix A).

6 Die übrigen Quotengruppen waren „Männer, 30-49 Jahre“ (Zielgröße: $n=28$ ), „Männer, ab 50 Jahre“ $(\mathrm{n}=21)$, „Frauen, 18-29 Jahre“ $(\mathrm{n}=63)$, „Frauen, 30-49 Jahre“ $(\mathrm{n}=28)$ und „Frauen, ab 50 Jahre“ $(\mathrm{n}=28)$.

7 Ein Ersatz-Proband (32 Jahre) lag im Alter knapp über der vorgesehenen Quote (18-29 Jahre). 
sieben Gruppen unterschieden sich nicht signifikant nach Alter, Geschlecht, Schulbildung, Konfession oder politischer Grundhaltung. ${ }^{8}$

Tabelle 1: Geplante Verteilung der Quotengruppen auf die Experimentalbedingungen („Brutto“) und empirische Verteilung nach Probanden-Ausfällen („Netto“)

\begin{tabular}{llccccccc}
\hline „Brutto“ & & EG 1 & EG 2 & EG 3 & EG 4 & EG 5 & EG 6 & KG \\
\hline Alter & Geschlecht & $n$ & $n$ & $n$ & $n$ & $n$ & $n$ & $n$ \\
18-29 J. & Männer & 7 & 7 & 7 & 7 & 7 & 7 & 7 \\
& Frauen & 9 & 9 & 9 & 9 & 9 & 9 & 9 \\
30-49 J. & Männer & 4 & 4 & 4 & 4 & 4 & 4 & 4 \\
& Frauen & 4 & 4 & 4 & 4 & 4 & 4 & 4 \\
Ab 50 J. & Männer & 3 & 3 & 3 & 3 & 3 & 3 & 3 \\
& Frauen & 4 & 4 & 4 & 4 & 4 & 4 & 4 \\
\hline Gesamt & & 31 & 31 & 31 & 31 & 31 & 31 & 31 \\
\hline
\end{tabular}

\begin{tabular}{llccccccc}
\hline „Netto“ & & EG 1 & EG 2 & EG 3 & EG 4 & EG 5 & EG 6 & KG \\
\hline Alter & Geschlecht & $n$ & $n$ & $n$ & $n$ & $n$ & $n$ & $n$ \\
18-29 J. & Männer & 8 & 7 & 6 & 5 & 7 & 6 & 7 \\
& Frauen & 8 & 9 & 8 & 8 & 7 & 8 & 9 \\
30-49 J. & Männer & 3 & 3 & 4 & 2 & 4 & 4 & 3 \\
& Frauen & 3 & 3 & 4 & 4 & 4 & 4 & 3 \\
Ab 50 J. & Männer & 2 & 2 & 1 & 4 & 3 & 1 & 2 \\
& Frauen & 6 & 3 & 3 & 3 & 3 & 4 & 5 \\
\hline Gesamt & & 30 & 27 & 26 & 26 & 28 & 27 & 29 \\
\hline
\end{tabular}

Nach Einstiegsfragen ermittelte der Fragebogen zunächst die Werte-Relevanz der Probanden. ${ }^{9}$ Unter neun Werten waren auch jene, die als Frame im Artikel fungierten. Die Probanden sollten alle Werte in eine Rangreihe bringen. Danach folgten der Artikel sowie sechs Pufferfragen zu dessen Qualität und zur Mediennutzung. Danach sollten die Probanden alles aufschreiben, was ihnen zur EU-Erweiterung in den Sinn kam, um den jeweiligen Frame des Artikels nochmals zu aktivieren. Der erste Fragenblock zu den abhängigen Variablen ermittelte, ob die Probanden den Wert und den Politiker aus dem Artikel kognitiv verknüpften. Dazu dienten zwei allgemeine und eine konkrete Frage. Die erste lautete: „Wer steht Ihrer Meinung nach für folgende Werte, [...] Merkel oder [...] Steinmeier?“. Unter den neun Werten waren auch Menschenrechte, internationale Sicherheit und wirtschaftliche Stabilität. Die zweite Frage lautete: „Manche politischen Ziele verbinden wir ja mit ganz bestimmten Politikern. Wie ist das mit den folgenden Zielen, verbinden Sie diese politischen Ziele mit [...] Merkel oder mit [...] Steinmeier?“. Diese Ziele (z. B. „Stärkere Achtung der Menschenrechte“) entsprachen den neun Wer-

8 Alter: Chi $^{2}=3.16 ; \mathrm{df}=12 ; \mathrm{p}>.99 ;$ Geschlecht: Chi $^{2}=.66 ; \mathrm{df}=6 ; \mathrm{p}>.99$; Schulbildung: Chi $^{2}=$ $4.03 ; \mathrm{df}=6 ; \mathrm{p}>.67 ;$ Konfession: $\mathrm{Chi}^{2}=5.45 ; \mathrm{df}=6 ; \mathrm{p}>$.48; Politische Grundhaltung: $\mathrm{F}=.72$; $\mathrm{df}=6 ; \mathrm{p}>.63($ vgl. Tabelle 1$)$.

9 Die neun Werte entstammen weitgehend einer Auflistung bei Fuchs/Klingemann (1989: 489-490). Die Abfrage mit einer Rangskala folgt Rokeach (1973: 27; zit. n. Keany/Glueckauf 1999: 147-148). Vergleichbar gingen z. B. Nelson/Oxley (1999: 1046) vor. 
ten. Für die Auswertung wurde die jeweils bipolare Skala von 1 (Merkel) bis 6 (Steinmeier $)^{10}$ in eine personenunabhängige Skala von 1 (keine Verknüpfung zwischen dem Wert und dem Politiker aus dem Artikel in Relation zum anderen Politiker) bis 6 (sehr starke Verknüpfung) recodiert. Das war notwendig, um einen personenunabhängigen Haupteffekt der Werte-Rahmung ermitteln zu können. Ansonsten hätten sich die Mittelwerte für beide Politiker bei der Varianzanalyse wechselseitig aufgehoben. Hier mag man einwenden: Statt der relativen Abfrage mit beiden Politikern als Pole hätte man auch für jeden Politiker jeweils unipolar abfragen können. Das hätte aber den Fragebogen länger gemacht. Vor allem aber wäre die Antwort für den zweiten Politiker immer von der Antwort für den ersten Politiker abhängig gewesen - und das wäre kaum anders als eine relative Abfrage. Die dritte Frage stellte drei fiktive EU-Gipfeltreffen vor, die sich um Ereignisse drehten, die jeweils einen der drei Werte tangierten. Die Probanden sollte für Merkel und für Steinmeier angeben, bei welchem Gipfel sich der Politiker ihrer Meinung nach engagiert hatte. ${ }^{11}$

Der zweite Block erfasste Indikatoren für Priming-Effekte. Die Fragen zu Image und Direktwahl der Kandidaten folgten weitgehend der Formulierung im Politbarometer (West) 2005 (ZA 4259) bzw. der Vorwahlstudie 1994 (ZA 2599). ${ }^{12}$ Die Skala entsprach aber den allgemeinen Fragen zur Verknüpfung von Wert und Politiker. Die Imagemerkmale wurde zu einem Index gebündelt (Cronbachs Alpha .92), der Werte von 1 (Politiker besitzt keines der Merkmale) bis 6 (alle Merkmale) annehmen konnte. Die recodierte Skala zur Direktwahl reichte von 1 (Politiker definitiv nicht wählen) bis 6 (definitiv wählen). Die Frageformulierung „Und wer hat Ihrer Meinung nach mehr Rückhalt in der eigenen Partei [...]?“ griff eine ähnliche Frage aus dem Politbarometer (West) 2005 auf (V172). Die recodierte Skala reichte von 1 (Politiker hat gar keinen Parteirückhalt) bis 6 (sehr starken Parteirückhalt). Zudem wurde die politische Grundhaltung auf einer bipolaren Skala von 1 (links) bis 7 (rechts) ermittelt. ${ }^{13}$ Den Abschluss bildeten soziodemographische Merkmale.

\section{Ergebnisse}

\subsection{Verknüpfungseffekte}

Wenn Medien einen Politiker in den Rahmen eines Wertes stellen, verbinden Rezipienten den Politiker mit diesem Wert stärker als bei einer anderen Rahmung. Bei der ersten Frage zu dieser kognitiven Verknüpfung sind die Befunde konform mit H1: Stellte der Artikel Merkel bzw. Steinmeier in den Rahmen internationale Sicherbeit, dann verknüpften die Probanden den jeweiligen Politiker stärker mit internationaler Sicherheit

$10 \mathrm{Zu}$ dieser Form der relativen Frage vgl. z. B. Kepplinger et al. (1994: 95) oder Brettschneider (2002: 94-95).

11 EU-Gipfeltreffen „zum Georgien-Konflikt und dessen Auswirkungen auf die internationale Sicherheit“, „zur internationalen Finanzkrise und deren Folgen für die EU-Mitgliedsstaaten“ und „zur weltweiten Verletzung von Menschenrechten und zu politischer Unterdrückung".

12 Die Primärforscher bei ZA 4259 sind M. Berger, M. Jung, D. Roth (Forschungsgruppe Wahlen). Die Primärforscher bei ZA 2599 sind M. Berger, M. Jung, D. Roth (IPOS, Mannheim) sowie W. G. Gibowski (Bundespresseamt). Aus Platzgründen wird die jeweilige Nummer des Zentralarchivs für empirische Sozialforschung an der Universität Köln zitiert. Vgl. www.gesis.org/dienstleistungen/daten/umfragedaten/politbarometer/studienuebersicht [19.02.2010]. Ein Merkmal ist aus Kepplinger et al. (1994) entnommen.

13 Damit wurde eine ähnliche Frage aus der Vorwahlstudie 1994 (ZA 2599; V169) aufgegriffen. 
$(\mathrm{M}=4.31$ bzw. $\mathrm{M}=3.96)$ als bei einer Rahmung über Menschenrechte $(\mathrm{M}=3.60 \mathrm{bzw}$. $\mathrm{M}=3.69)$ oder wirtschaftliche Stabilität $(\mathrm{M}=3.54 \mathrm{bzw} . \mathrm{M}=3.11) \cdot{ }^{14}$ Für die Verknüpfung mit wirtschaftlicher Stabilität zeigte sich nur ein Politiker-Effekt: Merkel wurde damit durchweg stärker in Verbindung gebracht. Für Menschenrechte gab es keine Gruppenunterschiede. Das bestätigt H2, wonach der Verknüpfungseffekt für Werte, die größere Bedeutung für die meisten Menschen haben, stärker ausfällt als für Werte mit geringerer Bedeutung für die meisten Menschen.

Der Befund für internationale Sicherheit bestätigte sich für Probanden, die diesem Wert subjektiv hohe Relevanz zuschrieben. ${ }^{15}$ Bei geringer Relevanz war die Verknüpfung zwischen Politiker und Wert schwächer. Dennoch blieb der Unterschied zwischen Lesern des Artikels, der Merkel in den Rahmen von Sicherheit stellte $(M=4.17)$, und Lesern der anderen Artikel $(M=3.12$ bzw. $M=3.64)$ bestehen. Für Steinmeier gab es keine Unterschiede. Damit kann F1 zur subjektiven Werte-Relevanz noch nicht eindeutig beantwortet werden. Als weitere Moderatorvariable wurde die politische Grundbaltung berücksichtigt (vgl. Abbildung 1). Unter den Probanden mit „rechter“ Grundhaltung (Mediansplit) verknüpften die Leser des Artikels, der Merkel in den Rahmen von Menschenrechten stellte, die Kanzlerin stärker mit diesem Wert $(\mathrm{M}=4.91)$, als die Leser der Artikel, die Merkel aus der Perspektive von Sicherheit ( $M=3.77)$ oder wirtschaftlicher Stabilität $(\mathrm{M}=3.79)$ thematisierten. Wie erwartet, war der Verknüpfungseffekt bei Menschenrechtsfragen in der Türkei weniger stark als bei internationaler Sicherheit. Die politische Grundhaltung hat das aber in gewisser Hinsicht kompensiert. Unter den „rechten“ Probanden verknüpften die Leser des Artikels, der Steinmeier in den Rahmen internationaler Sicherbeit stellte, diesen Politiker stärker mit Sicherheit (M $=4.25)$ als Leser der anderen Artikel $(M=3.25$ bzw. $M=2.92)$. Warum eine „rechte“ Grundhaltung ausgerechnet beim SPD-Politiker, nicht aber bei der Unionspolitikerin verstärkend wirkte, lässt sich erklären, wenn man die Kontrollgruppe $(n=29)$ heranzieht: Unter diesen Probanden stand eher Merkel $(\mathrm{M}=2.66$; Skala von $1=$ Merkel bis $6=$ Steinmeier) für Menschenrechte. Mit internationaler Sicherheit wurde jedoch eher der Außenminister verknüpft $(\mathrm{M}=3.38) .{ }^{16}$ Damit bestätigte sich $\mathrm{H} 3$ zunächst nur teilweise.

Die zweite allgemeine Frage zum Verknüpfungseffekt zielte nur vordergründig auf politische Ziele und war eine Kontrollfrage. Die bisherigen Befunde bestätigten sich weitgehend. Bei hoher Relevanzzuschreibung verknüpften z. B. die Leser des Artikels, der Merkel bzw. Steinmeier in den Rahmen von Sicherheit stellte, den jeweiligen Politiker stärker mit dem politischen Ziel, Stärkere internationale Sicherbeit “ $(\mathrm{M}=4.75 \mathrm{bzw}$. $\mathrm{M}=4.18)$ als Leser der Artikel, die mit Menschenrechten $(\mathrm{M}=3.86$ bzw. $\mathrm{M}=3.72)$ oder wirtschaftlicher Stabilität $(\mathrm{M}=3.18$ bzw. $\mathrm{M}=3.63)$ argumentieren. ${ }^{17}$ Auch die in $\mathrm{Ab}-$

14 Levene-Test auf Varianzgleichheit: $\mathrm{F}=.66$; df1 = 5; df2 = 151; n.s. ( $\mathrm{p}>.64)$. Erklärte Varianz: $4 \%$. Haupteffekt „Werte-Frame“: $F=4.46 ; \mathrm{df}=2 ; \mathrm{p}<.05$. Der Post-hoc-Test (Scheffé) fasste (a) Menschenrechte und Sicherheit sowie (b) Menschenrechte und Wirtschaftliche Stabilität zusammen.

15 Hohe (niedrige) Relevanz entspricht einem Rangplatz $\geq(<)$ Modus.

16 Für Probanden mit „linker“ Grundhaltung zeigten sich keine Haupteffekte der Rahmung. Der erwähnte Politiker-Effekt für wirtschaftliche Stabilität bestätigte sich hier aber ebenfalls.

17 Levene-Test auf Varianzgleichheit: $\mathrm{F}=.45$; df1 $=5$; df2 $=72 ; \mathrm{p}>.81$. Erklärte Varianz: $3 \%$. Haupteffekt „Werte-Frame“: $F=3.41 ; \mathrm{df}=2 ; \mathrm{p}<.05$. Der Post-hoc-Test (Scheffé) fasste (a) Menschenrechte und Sicherheit sowie (b) Menschenrechte und Wirtschaftliche Stabilität zusammen. 
Abbildung 1: Verknüpfung des Werts „Internationale Sicherbeit“ bzw. "Menschenrechte" und des Politikers aus dem Artikel (Mittelwerte)Probanden mit "rechter" politischer Grundhaltung"

a) Verknüpfung von Politiker und Wert „Internationale Sicherheit““ bei ,rechter" politischer Grundhaltung

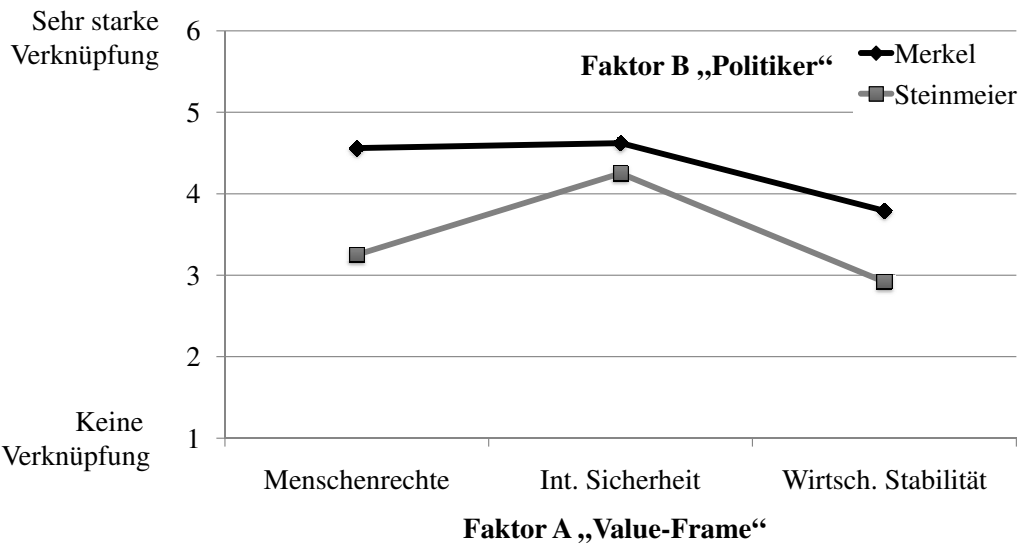

b) Verknüpfung von Politiker und Wert „Menschenrechte“ bei ,rechter" politischer Grundhaltung

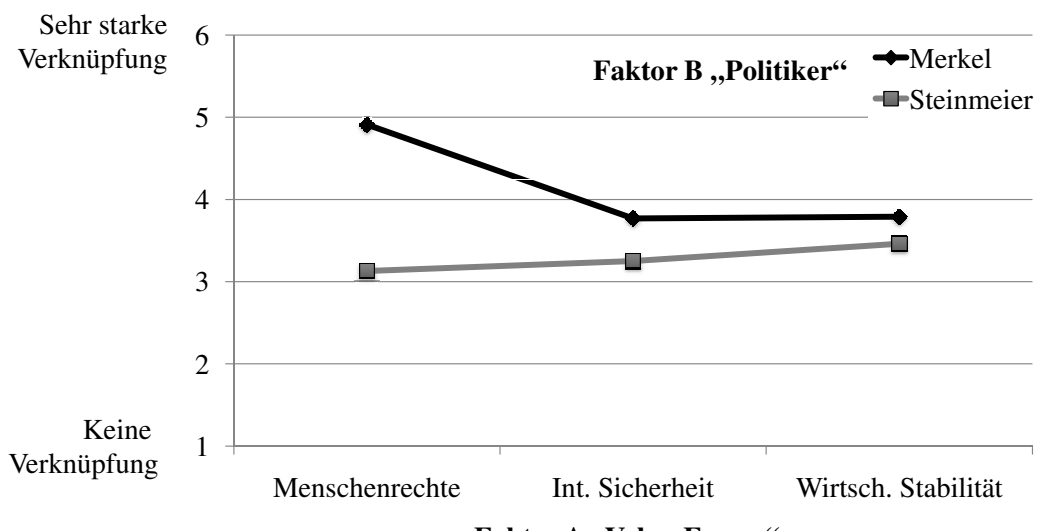

Faktor A, ,Value-Frame“

a) Zweifaktorielle Varianzanalyse für „Internationale Sicherheit“

Levene-Test auf Varianzgleichheit: $\mathrm{F}=.23$; df1 $=5$; df2 $=65 ; \mathrm{p}>.94$; Erklärte Varianz: $10 \%$ Haupteffekt „Werte-Frame“: $\mathrm{F}=3.38 ; \mathrm{df}=2 ; \mathrm{p}<.05$

Haupteffekt „Politiker“: $\mathrm{F}=5.45 ; \mathrm{df}=1 ; \mathrm{p}<.05$

Post-hoc-Test (Scheffé) fasst knapp ( $\mathrm{p}=.06)$ alle drei Gruppen zu einer zusammen.

b) Zweifaktorielle Varianzanalyse für „Menschenrechte“

Levene-Test auf Varianzgleichheit: $\mathrm{F}=.61 ; \mathrm{df} 1=5$; df2 = 65; $\mathrm{p}>$.69; Erklärte Varianz: $9 \%$ Haupteffekt „Politiker“: F = 7.02; df =1; $\mathrm{p}<.05$

* Hinweise: Die Aufteilung erfolgte anhand des Median = 3: Ein Wert $\leq(>)$ Median entspricht auf der Skala von 1 (links) bis 7 (rechts) einer „linken“ („rechten“) politischen Grundhaltung. 36 
bildung 1 dargestellten Befunde bestätigten sich. ${ }^{18}$ Das legt einen Konsistenzeffekt nahe. Zwar wurden beide Fragen durch eine längere Pufferfrage im Fragebogen getrennt. Für "Menschenrechte“ $(r=.77 ; p<.001)$ und "Wirtschaftliche Stabilität“ $(r=.82 ; p<.001)$ korrelierten die Antworten aber jeweils stark. Die Korrelation für „Sicherheit“ war geringer $(\mathrm{r}=.65 ; \mathrm{p}<.001)$.

Die dritte, konkretere Frage zum Verknüpfungseffekt stellte drei fiktive EU-Gipfeltreffen vor, die sich um Ereignisse drehten, die jeweils einen der drei Werte tangierten. Die Probanden sollten angeben, bei welchem Gipfel sich Merkel und bei welchem sich Steinmeier ihrer Meinung nach engagiert hatte. Die Antworten für jenen Politiker, der nicht im jeweiligen Artikel vorkam, wurden bei der Auswertung nicht berücksichtigt. Für Merkel zeigt sich zunächst ein Deckeneffekt (vgl. Abbildung 2, obere Hälfte): Etwa drei Viertel der Leser eines Artikels, in dem Merkel vorkam, glaubten an ein Engagement der Kanzlerin beim Gipfel zur Finanzkrise - ungeachtet der Artikel-Rahmung. Das widerspricht H1. Aber ein schwacher Effekt ist damit umso bedeutsamer: Leser des Artikels, der Merkel in den Rahmen von internationaler Sicherheit stellte (vgl. Kuchendiagramm in der Mitte), vermuteten etwas häufiger ein Engagement der Kanzlerin beim „EU-Gipfeltreffen zum Georgien-Konflikt und dessen Auswirkungen auf die internationale Sicherheit“ (19\%) als Leser des Artikels, der mit Menschenrechten (10\%) bzw. wirtschaftlicher Stabilität (12\%) argumentierte (vgl. schwarze „Kuchenstücke“ in Abbildung 2). Konform mit H2 zeigte sich kein vergleichbarer Effekt für die Rahmung Merkels im Lichte von Menschenrechten bzw. wirtschaftlicher Stabilität (vgl. dunkelund hellgraue „Kuchenstücke“).

Anders als H2 vermutete, zeigte sich der Verknüpfungseffekt für Steinmeier mehr oder minder für alle drei Value-Frames (vgl. Abbildung 2, untere Hälfte): Erstens vermuteten Leser des Artikels, der Steinmeier in den Rahmen von Menschenrechten stellte, häufiger ein Engagement Steinmeiers beim „EU-Gipfeltreffen zur weltweiten Verletzung von Menschenrechten und zu politischer Unterdrückung“ (46\%) als Leser der anderen Artikel (26\% bzw. $20 \%$ ). Zweitens glaubten Leser des Artikels, der aus der Warte von internationaler Sicherbeit argumentierte, häufiger an ein Engagement Steinmeiers beim Sicherheitsgipfel (52\%) als Leser des Artikels, der ihn in den Rahmen von Menschenrechten stellte (39\%). Allerdings war dieser Anteil bei Lesern des wirtschaftlich argumentierenden Artikels ebenso hoch (52\%). Drittens vermuteten Leser eines Artikels mit wirtschaftlicher Rahmung nur etwas häufiger (28\%) ein Engagement des SPD-Manns beim Gipfeltreffen zur Finanzkrise (28\%) als Leser der anderen Artikel (15\% bzw. $22 \%) .{ }^{19}$ Diese Befunde widersprechen H2 sowie den bisherigen Ergebnissen, wonach der Verknüpfungseffekt bei internationaler Sicherheit stärker ist als bei den anderen Value-Frames. Allerdings sind alle Indikatorfragen für den Verknüpfungseffekt zu betrachten. Denn in der Gesamtschau war der Verknüpfungseffekt nur für internationale Sicherheit bei allen drei Indikatorfragen sowie beiden Politikern zu beobachten.

Für den Filter der persönlichen Werte-Relevanz ist zweierlei festzuhalten: Erstens bestätigten sich die Befunde für Steinmeier bei hoher wie niedriger Werte-Relevanz. Die Gruppenunterschiede waren aber bei geringer Relevanzzuschreibung durchweg deutli-

18 Aber der Framing-Effekt war jeweils nicht signifikant. Für „Menschenrechte“ ergab sich ein Politiker-Effekt $(\mathrm{F}=4.90 ; \mathrm{df}=1 ; \mathrm{p}<.05)$; Levene-Test auf Varianzgleichheit: $\mathrm{F}=3.26$; df1 = $5 ; \mathrm{df} 2=64 ; \mathrm{p}>.86$.

19 Wegen nominalem Skalenniveau wurden drei Chi²-Tests (für drei Frames, für zwei Politiker und für sechs Frame-Politiker-Kombinationen) durchgeführt. Die „Interaktion“ zwischen Value-Frame und Politiker kam zustande, weil es bei Merkel nur für einen, bei Steinmeier aber für alle drei Werte einen Framing-Effekt gab. 
Abbildung 2: Situationsspezifische Verknüpfung des Wertes und Politikers aus dem Artikel* (Anteile in Prozent)

Merkel hat sich engagiert bei ...

EU-Gipfel zu Menschenrechten

EU-Gipfel zu internationaler Sicherheit

EU-Gipfel zu Finanzkrise

\section{Faktor A, ,Value-Frame“}

Menschenrechte

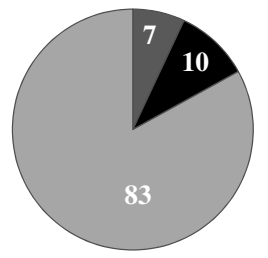

Int. Sicherheit

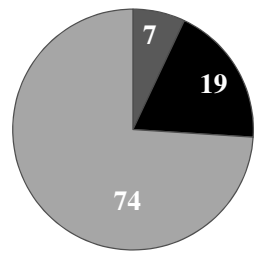

Wirt. Stabilität

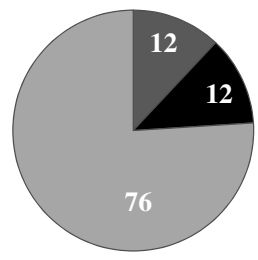

Steinmeier hat sich engagiert bei ...

EU-Gipfel zu Menschenrechten

EU-Gipfel zu internationaler Sicherheit

EU-Gipfel zu Finanzkrise

\section{Faktor A, „Value-Frame“}

Menschenrechte

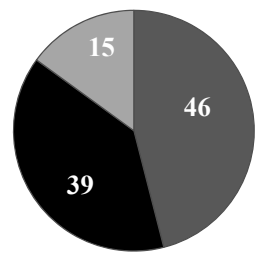

Int. Sicherheit

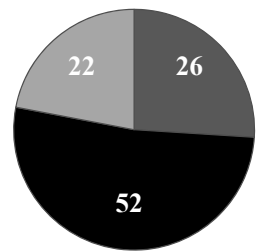

Wirt. Stabilität

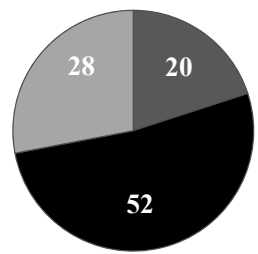

"Die Antworten der Probanden für jenen Politiker, der jeweils nicht im Artikel vorkam, wurden bei den $\mathrm{Chi}^{2}$-Tests nicht berücksichtigt.

„Haupteffekt“ Werte-Frame: $\mathrm{Chi}^{2}=2,92 ; \mathrm{df}=4$; n.s.

„Haupteffekt“ Politiker: $\mathrm{Chi}^{2}=50,61 ; \mathrm{df}=2 ; \mathrm{p}<0,001$

„Interaktionseffekt“ Werte-Frame * Politiker: Chi $^{2}=57,92 ; \mathrm{df}=10 ; \mathrm{p}<0,001$

cher. Zweitens war dies bei den beiden allgemeinen Fragen genau umgekehrt. Das legt nahe, dass die konkrete Frage nach den Gipfeltreffen anschaulicher war. Möglicherweise kommt sie Probanden entgegen, für die ein Wert weniger wichtig ist und die sich bislang kaum Gedanken dazu gemacht haben. Die abstrakteren Fragen verlangen dagegen, dass sich Menschen schon Gedanken über die jeweiligen Werte gemacht haben. Und Gedanken macht man sich nur über etwas, was man für relevant hält-und umgekehrt. Jedenfalls bleiben die bisherigen Befunde zur persönlichen Werte-Relevanz (vgl. z. B. Shen/Ed- 
wards 2005; Slothuus 2008) weiterhin widersprüchlich. Offenkundig spielt jedoch die Frageformulierung eine gewisse Rolle.

Der Filter der politischen Grundhaltung wirkte weitgehend verstärkend. Unter Probanden mit „linker“ Grundhaltung (Mediansplit) bestätigen sich die für Steinmeier beschriebenen Befunde. Vor allem glaubten unter den Probanden mit „rechter“ Grundhaltung die Leser des Artikels, der Merkel in den Rahmen von Sicherheit stellte, sogar häufiger an ein Engagement der Kanzlerin beim Gipfel zur Georgien-Krise (23 \%) als Leser der anderen Artikel (9\% bzw. 15 \%). Umgekehrt war dergleichen bei Probanden aus dem jeweils gegnerischen Lager nicht erkennbar. Nur in einem Fall kam eine „rechte“ Grundhaltung dem SPD-Politiker zugute. Denn „rechte“ Leser des Artikels, der Steinmeier in den Rahmen von Menschenrechten stellte, glaubten häufiger an ein Engagement beim Gipfel zu Menschenrechten (75\%) als Leser der anderen Artikel (18\% bzw. $25 \%$ ). Damit bestätigte sich $\mathrm{H} 3$ erneut nur teilweise.

\subsection{Priming-Effekte}

Der Value-Frame kann als Prime für Urteile über Politiker fungieren: Da der Zeitungsartikel den jeweiligen Politiker in allen Artikelversionen durchweg als „Garant“ für den betreffenden Wert präsentierte, dürften Rezipienten den Politiker positiver beurteilen als ohne solches Value-Framing. Da der Verknüpfungseffekt in der Gesamtschau bei der Rahmung über internationale Sicherbeit stärker war als bei den beiden anderen ValueFrames, sollte das Urteil über Merkel bzw. Steinmeier bei dieser Rahmung auch positiver sein als in den beiden anderen Fällen. Für das Politikerimage zeigte sich aber nur ein Politiker-Effekt: Leser eines der Artikel über Merkel hatten ein besseres Bild von ihr (M = 3.98) als Leser eines der Artikel über Steinmeier $(\mathrm{M}=2.89) \cdot{ }^{20}$ Nur teilweise konform mit H4 sind die Befunde zur Direktwahlfrage: Zwar wünschten sich die Probanden im Durchschnitt generell eher Merkel als Steinmeier. ${ }^{21}$ Allerdings wollten Leser des Artikels, der Merkel in den Rahmen internationaler Sicherheit stellte, die Politikerin - im Vergleich zu Steinmeier - noch häufiger direkt wählen $(M=5.04)$ als Leser der Artikel, die Merkel in den Rahmen von Menschenrechtsfragen bzw. wirtschaftlicher Stabilität stellten $(M=4.37$ bzw. $M=4.40)$. Mit H4 wäre der gleiche Befund für Steinmeier zu erwarten. Allerdings mündete die Rahmung als Garant für internationale Sicherheit nicht in einem positiveren Urteil - im Gegenteil: Leser des Artikels, der Steinmeier aus dieser Perspektive thematisierte, wollten ihn noch seltener im Kanzleramt sehen $(M=2.33)$ als Leser der anderen Artikel über ihn $(\mathrm{M}=3.00 \mathrm{bzw} . \mathrm{M}=2.74){ }^{22}$ Einen vergleichbaren Interaktionseffekt gab es für den von den Probanden vermuteten Parteirückhalt des jeweiligen Politikers aus dem Artikel. ${ }^{23}$ Möglicherweise lässt sich die „Schere“ zwischen Merkel und Steinmeier damit erklären, dass die Darstellung als „Garant“ für internationale Sicherheit nur bei einem "passenden“ Näbrboden im Meinungsklima in einem po-

20 Levene-Test auf Varianzgleichheit: $\mathrm{F}=1.31$; df1 $=5$; df2 $=153$; p >.26. Erklärte Varianz: $36 \%$. Haupteffekt „Politiker“: F = 85.26; df =1; $\mathrm{p}<.001$.

21 Unter den Probanden der Kontrollgruppe (Experimentalgruppen) betrug der Mittelwert $\mathrm{M}=$ $2.53(\mathrm{M}=2,55)$ auf der ursprünglichen Skala von 1 (Merkel) bis 6 (Steinmeier).

22 Levene-Test auf Varianzgleichheit: $\mathrm{F}=1.00 ; \mathrm{df} 1=5$; df2 $=155 ; \mathrm{p}>.42$. Erklärte Varianz: $28 \%$. Haupteffekt „Politiker“: $\mathrm{F}=62.16$; $\mathrm{df}=1 ; \mathrm{p}<.001$; Interaktionseffekt „Werte-Frame/ Politiker": $\mathrm{F}=2.82 ; \mathrm{df}=2 ; \mathrm{p}<.06$.

23 Levene-Test auf Varianzgleichheit: $\mathrm{F}=1.07$; df1 = 5; df2 = 157; $\mathrm{p}>$.37. Erklärte Varianz: $45 \%$. Haupteffekt „Politiker“: $\mathrm{F}=128.14 ; \mathrm{df}=1 ; \mathrm{p}<.001$. Interaktionseffekt „Werte-Frame/ Politiker“: $\mathrm{F}=3.48 ; \mathrm{df}=2 ; \mathrm{p}<.05$. 
sitiveren Urteil über den jeweiligen Politiker mündet. Dass das Meinungsklima für Merkel weit günstiger war als für Steinmeier, zeigen Repräsentativumfragen aus dem Erhebungszeitraum des Experiments. ${ }^{24}$

Tabelle 2: Primingeffekte auf wabrgenommenes Image, Direktwablabsicht und Urteil über den Parteirückhalt des jeweiligen Politikers (Mittelwerte) - Probanden mit „rechter" politischer Grundhaltung

\begin{tabular}{lccccccc}
\hline & \multicolumn{3}{c}{ Menschenrechte } & \multicolumn{2}{c}{$\begin{array}{c}\text { nnternationale } \\
\text { Sicherheit }\end{array}$} & \multicolumn{2}{c}{$\begin{array}{c}\text { Wirtschaftliche } \\
\text { Stabilität }\end{array}$} \\
\hline & \multicolumn{3}{c}{$\begin{array}{c}\text { Merkel Steinmeier } \\
(\mathrm{n}=30)\end{array}$} & $\begin{array}{c}\text { Merkel } \\
(\mathrm{n}=26)\end{array}$ & $\begin{array}{c}\text { Steinmeier } \\
(\mathrm{n}=27)\end{array}$ & $\begin{array}{c}\text { Merkel } \\
(\mathrm{n}=28)\end{array}$ & $\begin{array}{c}\text { Steinmeier } \\
(\mathrm{n}=27)\end{array}$ \\
„Rechte“ Grundhaltung & $\mathrm{M}$ & $\mathrm{M}$ & $\mathrm{M}$ & $\mathrm{M}$ & $\mathrm{M}$ & $\mathrm{M}$ \\
(> Median) & $(\mathrm{SD})$ & $(\mathrm{SD})$ & $(\mathrm{SD})$ & $(\mathrm{SD})$ & $(\mathrm{SD})$ & $(\mathrm{SD})$ \\
\hline Image-Index A & 4.83 & 2.53 & 4.88 & 2.08 & 4.24 & 2.80 \\
& $(.94)$ & $(.49)$ & $(1.00)$ & $(.92)$ & $(.69)$ & $(1.00)$ \\
Direktwahl zum Bundeskanzler & B & 5.27 & 1.88 & 5.69 & 1.67 & 4.93 & 2.46 \\
& $(1.19)$ & $(.99)$ & $(.63)$ & $(.98)$ & $(1.00)$ & $(1.51)$ \\
Rückhalt in der eigenen Partei C & 4.91 & 2.38 & 5.08 & 1.75 & 4.71 & 2.46 \\
& $(1.14)$ & $(1.06)$ & $(.76)$ & $(.75)$ & $(1.07)$ & $(1.39)$ \\
\hline
\end{tabular}

Jeweils zweifaktorielle Varianzanalysen:

A Levene-Test auf Varianzgleichheit: $\mathrm{F}=1.23$; df1 = 5; df2 = 64; $\mathrm{p}>$.30; Erklärte Varianz: $61 \%$

Haupteffekt „Politiker“: $\mathrm{F}=101.42 ; \mathrm{df}=1 ; \mathrm{p}<.001$

Interaktionseffekt „Werte-Frame/Politiker“: $\mathrm{F}=3.91 ; \mathrm{df}=2 ; \mathrm{p}<.05$

B Levene-Test auf Varianzgleichheit: $\mathrm{F}=1.74$; df1 = 5; df2 = 65; $\mathrm{p}>.13$; Erklärte Varianz: $70 \%$

Haupteffekt „Politiker“: F = 158.15; df = 1; $\mathrm{p}<.001$

Interaktionseffekt „Werte-Frame * Politiker“: $\mathrm{F}=3.39 ; \mathrm{df}=2 ; \mathrm{p}<.05$

${ }^{C}$ Levene-Test auf Varianzgleichheit: $\mathrm{F}=1.23 ; \mathrm{df} 1=5 ; \mathrm{df} 2=65 ; \mathrm{p}>.30$; Erklärte Varianz: $62 \%$

Haupteffekt „Politiker“: F = 113.24; df = 1; $\mathrm{p}<.001$

Die bislang beschriebene Rolle der Werte-Relevanz bestätigte sich beim Priming-Effekt vor allem für Steinmeier. Allerdings war der Interaktionseffekt aus Value-Frame und Politiker bei der Image- und der Direktwahlfrage (knapp) nicht signifikant. Die politische Grundhaltung der Probanden wirkte erneut verstärkend. So verschärfte sich unter „rechten“ Probanden der bereits beschriebene Priming-Effekt (vgl. Tabelle 2). Bei der Direktwahlfrage trat erneut ein Interaktionseffekt zwischen Value-Frame und Politiker auf, der wegen des Deckeneffekts für Merkel und des Bodeneffekts für Steinmeier umso bedeutsamer ist: Unter „rechten“ Probanden wollten Leser des Artikels, der Merkel in den Rahmen internationaler Sicherheit stellte, die Kanzlerin sogar noch häufiger wählen $(\mathrm{M}=5.69)$ als die Leser der anderen Artikel über sie $(M=5.27$ bzw. $M=4.93)$. Umgekehrt wünschten Leser des Artikels, der Steinmeier aus dieser Sicht rahmte, den Herausforderer sogar noch seltener im Kanzleramt $(M=1.67)$ als ohnehin schon die Leser der anderen Artikel über ihn ( $\mathrm{M}=1.88$ bzw. $\mathrm{M}=2.46)$.

24 Vgl. www.forschungsgruppewahlen.de/Umfragen_und_Publikationen/Politbarometer/Ar chiv/Politbarometer_2009/Juli_I [19.02.2010]. 


\section{Schlussfolgerungen}

Das Experiment zeigte erstens, dass die Rahmung eines Politikers als „Garant“ für einen politischen Wert bzw. ein politisches Ziel dazu führen kann, dass Rezipienten den Politiker mit diesem Wert bzw. Ziel verbinden. Ein solcher Verknüpfungseffekt ist anscheinend stärker bei Werten, die größere Bedeutung für die meisten Menschen haben. Zweitens gab es Hinweise auf Priming-Effekte, die aber offenbar durch das generelle Meinungsklima zu den beiden Politikern im Erhebungszeitraum der Studie, also im Bundestagswahlkampf 2009, gebrochen wurden. Die politische Grundhaltung der Probanden verstärkte die genannten Effekte in der Regel. Die Befunde zur moderierenden Rolle der persönlichen Werte-Relevanz waren dagegen widersprüchlich, was aber die Forschungslage widerspiegelt.

Die Studie unterliegt folgenden Beschränkungen: Erstens bleibt die Aussagekraft der Befunde notgedrungen auf das exemplarische Thema des Artikels beschränkt. Zweitens wurden Konsonanz und Kumulation als Wirkungsbedingungen theoretisch modelliert. Untersucht wurden aber streng genommen temporäre Effekte. Gerade deswegen aber sind die zunächst nicht sehr starken (signifikanten) Gruppenunterschiede durchaus nicht unbedeutsam. Drittens muss sich jedes Experiment auf wenige Faktoren beschränken. Für künftige Studien liegen z. B. zwei Fragen nahe: Zum einen dürfte es einen Unterschied machen, ob ein Politiker als "Garant" oder "Gefahr" für einen Wert präsentiert wird. Im zweiten Fall könnten die Effekte sogar deutlicher sein, wenn man die Forschung zu Negativismus berücksichtigt (vgl. z. B. Meffert et al. 2006). Zum anderen bietet sich eine Replikation für ein wahlkampfrelevantes Thema an.

Schließlich stellt sich die Frage, ob ein wertbezogenes Verständnis von Frames zu bisherigen Definitionen anschlussfähig ist. In seiner viel zitierten Definition benennt Entman (1993: 52) „problem definition, causal interpretation, moral evaluation and/or treatment recommendation" als Frame-Elemente. Value-Frames sind damit durchaus vereinbar. Erstens kann man sie als Kern der „moral evaluation“ begreifen. Zweitens wird der Blick durch Value-Framing nicht (nur) auf einen abstrakten Wert gelenkt, sondern auch auf bestimmte Ursachen oder Lösungen. So dürfte die Rahmung eines Krieges als Menschenrechtsfrage mit der Forderung nach einem humanitären UN-Einsatz statt eines Wirtschaftsboykotts einhergehen (vgl. auch B. Scheufele/Gasteiger 2007). Drittens ist Framing nur dann erfolgreich, wenn es an zentrale Werte und Grundsätze jener Gruppe andockt, die mobilisiert werden soll (vgl. Snow/Benford 1992: 141). Ähnlich argumentieren Gerhards/Neidhardt (1991: 72): „Läßt sich ein Problem in ein von den Bürgern geteiltes allgemeines Deutungsraster vom Wünschbaren, also in ein Wertemuster, einhaken, dann erhöht sich die Einsicht, daß es sich wirklich um ein Problem handelt“. Und auch Brewer (2002: 303) konstatiert: „[I]f frames influence how citizens link their values to issues, then political elites may be able to shape the public's understanding of political issues by disseminating value frames through the mass media“.

\section{Literatur}

Brettschneider, F. (2002). Spitzenkandidaten und Wahlerfolg. Personalisierung - Kompetenz Parteien. Ein internationaler Vergleich. Wiesbaden.

Brewer, P. R. (2002). Framing, Value Words, and Citizens` Explanations of their Issue Opinions, in: Political Communication 19, 303-316.

Brewer, P. R./Graf, J./Willnat, L. (2003). Priming or Framing. Media Influence on Attitudes toward Foreign Countries, in: Gazette 65, 493-508.

Brewer, P. R./Gross, K. (2005). Values, Framing, and Citizens' Thoughts about Policy Issues. Effects on Content and Quantity, in: Political Psychology 26, 929-948. 
Cappella, J. N./Jamieson, K. H. (1997). Spiral of Cynicism. The Press and the Public Good. New York, Oxford.

CDU/CSU (2009). Wir haben die Kraft. Gemeinsam für unser Land. Regierungsprogramm 2009-2013. Berlin.

DeVreese, C./Boomgaarden, J. (2003). Valenced News Frames and Public Support for the EU, in: Communications 28, 361-381.

Domke, D./Shah, D. V./Wackman, D. B. (1998). Media Priming Effects: Accessibility, Association, and Activation, in: International Journal of Public Opinion Research 10, 51-74.

Entman, R. M. (1993). Framing: Toward Clarification of a Fractured Paradigm, in: Journal of Communication 43, 51-58.

Fiske, S. T./Larter, D. R./Larter, W. M. (1983). The Novice and the Expert: Knowledge-based Strategies in Political Cognition, in: Journal of Experimental Social Psychology 19, 381-400.

Fuchs, D./Klingemann, H. D. (1989). Das Links-Rechts-Schema als politischer Code. Ein interkultureller Vergleich auf inhaltsanalytischer Grundlage, in: Haller, M./Hoffmann-Nowotny, H.-J./Zapf, W. (Hrsg.), Kultur und Gesellschaft. Frankfurt am Main/New York, 484-498.

Fuchs, D./Rohrschneider, R. (2005). War es dieses Mal nicht die Ökonomie? Der Einfluss von Sachfrageorientierungen auf die Wählerentscheidung bei der Bundestagswahl 2002, in: Falter, J. W./Gabriel, O. W./Weßels, B. (Hrsg.), Wahlen und Wähler. Analysen aus Anlass der Bundestagswahl 2002. Wiesbaden, 339-356.

Gabriel, O. W. (2009). Politische Kultur, in: Kaina, V./Römmele, A. (Hrsg.), Politische Soziologie. Ein Studienbuch. Wiesbaden, 17-51.

Gerhards, J./Neidhardt, F. (1991). Strukturen und Funktionen moderner Öffentlichkeit. Fragestellungen und Ansätze, in: Müller-Doohm, S./Neumann-Braun, K. (Hrsg.), Öffentlichkeit Kultur - Massenkommunikation. Beiträge zur Medien- und Kommunikationssoziologie. Oldenburg, 31-89.

Iyengar, S. (1991). Is Anyone Responsible? How Television Frames Political Issues. Chicago (Il.) Johnson-Laird, P. N. (1980). Mental Models in Cognitive Science, in: Cognitive Science 4, 71-115. Jung, Helmut (2005). Wertewandel und Wahlentscheidungen. Ergebnisse der empirischen Langzeitforschung. Eingangsstatement zum Panel „Was heute den Ausschlag gibt“ auf der internationalen Fachtagung „Politische Kommunikation in der globalisierten Welt - Richtungsentscheidungen in schwieriger Zeit“. Vortrag bei der Konrad-Adenauer-Stiftung, Mainz, 13.-14. Oktober 2005, www.kas.de/upload/veranstaltungen/15028/dokumente/Vortrag_131 005.ppt [19.02.2010].

Keany, K. C. M.-H./Glueckauf, R. L. (1999): Disability and Value Change: An Overview and Reanalysis of Acceptance of Loss Theory, in: Marinelli, R. P./Orto, A. E. D. (eds.), The Psychological and Social Impact of Disability. New York, 139-151.

Kepplinger, H. M./Brosius, H.-B./Dahlem, S. (1994). Wie das Fernsehen Wahlen beeinflußt. Theoretische Modelle und empirische Analysen. München.

Kepplinger, H. M./Brosius, H.-B./Staab, J. F. (1991). Opinion Formation in Mediated Conflicts and Crisis: A Theory of Cognitive-Affective Media Effects, in: International Journal of Public Opinion Research 3, 132-156.

Kevenhörster, P. (2006). Politikwissenschaft. Band 2: Ergebnisse und Wirkungen der Politik. Wiesbaden.

Kluckhohn, C. (1951). Values and Value Orientations in the Theory of Action. An Exploration in Definition and Classification, in: Parsons, T./Shils, E. A. (eds.), Toward a General Theory of Action. Cambridge, 388-433.

Korte, K.-R./Fröhlich, M. (2004). Politik und Regieren in Deutschland. Strukturen, Prozesse, Entscheidungen. 3. aktualisierte und überarbeitete Auflage. Paderborn u. a.

Matthes, J. (2007). Framing-Effekte. Zum Einfluss der Politikberichterstattung auf die Einstellungen der Rezipienten. München.

Meffert, M. M./Chung, S./Joiner, A. J./Waks, L./Garst, J. (2006): The Effects of Negativity and Motivated Information Processing during a Political Campagin, in: Journal of Communication $56,27-51$.

Nelson, T. E./ Oxley, Z. M. (1999). Issue Framing Effects on belief Importance and Opinion, in: The Journal of Politics 61, 1040-1067. 
Nelson, T. E./Clawson, R. A./Oxley, Z. M. (1997). Media Framing of Civil Liberties Conflict and its Effects on Tolerance, in: American Political Science Review 91, 567-583.

Nelson, T. E./Willey, E. (2001). Issue Frames that Strike a Value Balance: A Political Psychology Perspective, in: Reese, S. D./Gandy, O. H./Grant, A. (eds.), Framing Public Life. Perspectives on Media and our Understanding of the Social World. Mahwah (NJ), 245-265.

Neuman, W. R./Just, M. R./Crigler, A. N. (1992). Common knowledge. News and the Construction of Political Meaning. Chicago, London.

Operario, D./Fiske, S. T. (2004). Stereotypes. Content, Structures, Processes, and Context, in: Brewer, M. B./Hewstone, M. (eds.), Social Cognition. Malden (MA), 120-141.

Peter, J. (2002). Medien-Priming. Grundlagen, Befunde und Forschungstendenzen, in: Publizistik 47, 21-44.

Price, V./Nir, L./Cappella, J. N. (2005). Framing Public Discussion of Gay Civil Unions, in: Public Opinion Quarterly 69, 179-212.

Price, V./Tewksbury, D. (1997). News Values and Public Opinion: A Theoretical Account of Media Priming and Framing, in: Barett, G. A./Boster, F. J. (eds.), Progress in Communication Sciences: Advances in Persuasion. Vol. 13. Greenwich (CT), 173-212.

Reese, S. D. (2001): Prologue - Framing Public Life: A Bridging Model for Media Research, in: Reese, S. D./Gandy, O. H./Grant, A. (eds.), Framing Public Life. Perspectives on Media and our Understanding of the Social World. Mahwah (NJ), London, 7-31.

Rokeach, M. (1973). The Nature of Human Values. New York.

Rosenberg, M. J. (1956). Cognitive Structure and Attitudinal Affect, in: Journal of Abnormal Social Psychology 53, 367-372.

Scheufele, B. (2003). Frames-Framing - Framing-Effekte. Theoretische und methodische Grundlegung sowie empirische Befunde zur Nachrichtenproduktion. Wiesbaden.

Scheufele, B. (2004). Framing-Effects Approach: A Theoretical and Methodological Critique, in: Communications 29, 401-428.

Scheufele, B./Gasteiger, C. (2007). Berichterstattung, Emotionen und politische Legitimierung. Eine experimentelle Untersuchung zum Einfluss der Politikberichterstattung auf die Legitimierung politischer Entscheidungen am Beispiel von Bundeswehreinsätzen, in: Medien \& Kommunikationswissenschaft 55, 534-554.

Scheufele, B. T./Scheufele, D. A. (2009). Of Spreading Activation, Applicability and Schemas: Conceptual Distinctions and their Operational Implications for Measuring Frames and Framing Effects, in: D‘Angelo, P./Kuypers, J. (eds.), Doing News Framing Analysis: Empirical and Theoretical Perspectives. Hillsdale (NJ), 110-134.

Scheufele, D. A./Tewksbury, D. (2007). Framing, Agenda-Setting, and Priming: The Evolution of Three Media Effects Models, in: Journal of Communication 57, 9-20.

Schwartz, S. H. (1992). Universal in the Context and Structure of Values: Theoretical Advances and Empirical Tests in 20 Countries, in: Zanna, M. P. (eds.), Advances in Experimental Social Psychology. Vol. 25. San Diego (CA), 1-65.

Schwartz, S. H./Bardi, A. (2001). Value Hierarchies Across Cultures. Taking a Similarites Perspective, in: Journal of Cross-cultural Psychology 32, 268-290.

Semetko, H. A./Valkenburg, P. M. (2000). Framing European Politics: A Content Analysis of press and Television News, in: Journal of Communication 50, 93-109.

Shah, D. V./Domke, D./Wackman, D. B. (1996). „To thine own self be true“. Values, Framing, and Voter Decision-making Strategies, in: Communication Research 23, 509-560.

Shen, F./Edwards, H. H. (2005). Economic Individualism, Humanitarianism, and Welfare Reform. A Value-based Account of Framing Effects, in: Journal of Communication 55, 795-809.

Slothuus, R. (2008). More than Weighting Cognitive Importance: A Dual-Process Model of Issue Framing Effects, in: Political Psychology 29, 1-28.

Snow, D. A./Benford, R. D. (1992): Master Frames and Cycles of Protest, in: Morris, A. D./Mueller, C. M. (eds.), Frontiers in Social Movement Theory. New Haven (CT), 133-155.

SPD (2009). Sozial und demokratisch. Anpacken. Für Deutschland. Das Regierungsprogramm der SPD. Berlin. 


\section{Appendix A - Hintergrundmaterial zur Erstellung des Stimulus}

Bischoff, M. (2008/09). Die kontroversen Einstellungen in der EU zu einer türkischen Mitgliedschaft. Thesenpapier im Hauptseminar „Die EU-Nachbarschaftsbeziehungen“ (Prof. Dr. H. Hubel), Wintersemester 2008/09. Friedrich-Schiller-Universität. Jena.

Dietert, A. (2006). Menschen- und Minderheitenrechte: die Türkei und die europäischen Standards. Bundeszentrale für politische Bildung. Bonn, www.bpb.de/themen/35SQ8K,0,0,Men schen_und_Minderheitenrechte\%3A_die_T\%FCrkei_und_die_europ\%E4ischen_Standards .html [19.02.2010].

www.handelsblatt.com/politik/international/eu-osterweiterung-keine-zeit-fuerbremser;2259485 [19.02.2010].

www.tagesschau.de/ausland/meldung75740.html [19.02.2010].

www.spiegel.de/politik/deutschland/0,1518,626944,00.html [19.02.2010].

www.osmanischesreich.com/Geschichte/Literatur/Turkei/EU-Beitritt_der_Turkei/eu-beitritt _der_turkei.html [19.02.2010].

\section{Appendix B - Stimulusartikel am Beispiel „Angela Merkel“}

Menschenrechte
Türkei in die EU?
Merkel mabnt: „Mehr Men-
schenrechte“
BERLIN (bs) Am Montag traf
Bundeskanzlerin Angela Mer-
kel den türkischen Außenmi-
nister in Berlin. Dabei ging es
um die möglichen Beitrittsver-
handlungen der EU zur Türkei.
Merkel wies darauf hin, dass in
der Türkei noch vieles getan
werden müsse, bevor das Land
in die Europäische Union auf-
genommen werden könne. Da-
bei hob sie besonders „die Men-
schenrechtsfragen“ hervor.

Merkel: „Menschenrechte müssen eingebalten werden"

So seien die Kopenhagener Kriterien, wie zum Beispiel die Wahrung von Bürgerrechten und die Achtung von Minderheiten, von der Türkei bislang nicht erfüllt worden. Diese gelten jedoch als Voraussetzung für eine EU-Mitgliedschaft. „In den vergangenen Jahren hat sich die Menschenrechtslage in der Türkei nicht sichtbar verbessert", so die Bundeskanzlerin.

\begin{tabular}{|c|c|}
\hline Inter & Wir \\
\hline & \\
\hline $\begin{array}{l}\text { Merkel mahnt: "Meb } \\
\text { heit" }\end{array}$ & $\begin{array}{l}\text { Merkel mabnt: "Mebr } \\
\text { schaftsreformen" }\end{array}$ \\
\hline
\end{tabular}

BERLIN (bs) Am Montag traf Bundeskanzlerin Angela Merkel den türkischen Außenminister in Berlin. Dabei ging es um die möglichen Beitrittsverhandlungen der EU zur Türkei. Merkel wies darauf hin, dass in der Türkei noch vieles getan werden müsse, bevor das Land in die Europäische Union aufgenommen werden könne. Dabei hob sie besonders „die Sicherheitsproblematik" hervor.

Merkel: „Sicherheitsprobleme müssen gelöst werden"

So seien die Kopenhagener Kriterien, wie zum Beispiel eine sichere innenpolitische Lage und die Einhaltung der sicherheitspolitischen Kriterien (GASP) der EU, von der Türkei bislang nicht erfüllt worden. Diese gelten jedoch als Voraussetzung für eine EUMitgliedschaft. „In den vergangenen Jahren hat sich die Sicherheitspolitik der Türkei nicht sichtbar verbessert", so die Bundeskanzlerin.
BERLIN (bs) Am Montag traf Bundeskanzlerin Angela Merkel den türkischen Außenminister in Berlin. Dabei ging es um die möglichen Beitrittsverhandlungen der EU zur Türkei. Merkel wies darauf hin, dass in der Türkei noch vieles getan werden müsse, bevor das Land in die Europäische Union aufgenommen werden könne. Dabei hob sie besonders „die wirtschaftliche Stabilität" hervor.

Merkel: „Wirtschaftsreformen müssen umgesetzt werden"

So seien die Kopenhagener Kriterien, wie zum Beispiel eine funktionsfähige Marktwirtschaft und europäische Wettbewerbsfähigkeit, von der Türkei bislang nicht erfüllt worden. Diese gelten jedoch als Voraussetzung für eine EU-Mitgliedschaft. „In den vergangenen Jahren hat sich die wirtschaftliche Stabilität der Türkei nicht sichtbar verbessert", so die Bundeskanzlerin. 
Studie belegt Menschenrechtsverletzungen ken

belegt Sicherheitsrisi-

Eine aktuelle Studie im Auftrag der Menschenrechtsorganisation Amnesty International (aI) belegt, dass Folter und Unterdrückung immer noch Teil der Realität des Landes sind.

Trotz der Bemühungen der derzeitigen türkischen Regierung, solchen Missständen zu begegnen, sei die Umsetzung laut Merkel ungenügend. Angela Merkel erklärte, dass eine Aufnahme der Türkei einen menschenrechtlichen Rückschritt für die Europäische Union bedeuten würde: „Sowohl die Verfolgung aufgrund von politischen Meinungsäußerungen als auch die andauernde Praxis von Folter würden den Grundwerten der EU widersprechen.“ Obwohl die Türkei den EUBeitritt seit Jahren anstrebt, lehnt Merkel die Aufnahme ab.

Analog fürFrank-Walter-Steinmeier
Eine aktuelle Studie im Auf- Eine aktuelle Studie im Auftrag trag des Auswärtigen Amtes der Organisation für Wirtschaftbelegt, dass die Gefährdung liche Zusammenarbeit und Entdurch terroristische Gruppie- wicklung (OECD) belegt, dass rungen und innerpolitische Korruption und Misswirtschaft Spannungen immer noch Teil immer noch Teil der Realität des der Realität des Landes sind. Landes sind.

Trotz der Bemühungen der Trotz der Bemühungen der derderzeitigen türkischen Regie- zeitigen türkischen Regierung, rung, solchen Missständen zu solchen Missständen zu begegbegegnen, sei die Umsetzung nen, sei die Umsetzung laut Merlaut Merkel ungenügend. An- kel ungenügend. Angela Merkel gela Merkel erklärte, dass eine erklärte, dass eine Aufnahme der Aufnahme der Türkei die Si- Türkei die Europäische Union cherheit der EU gefährden wirtschaftlich überfordern würwürde: „Aufgrund der Grenz- de: „Die hohe Inflationsrate und verschiebung der EU müssten die Anpassungskosten der Wirtwir uns dann aktiv mit den au- schaft und Gesellschaft würden ßenpolitischen Konflikten des nicht nur den türkischen StaatsNahen Ostens und der arabi- haushalt, sondern dann auch die schen Nachbarn auseinander EU belasten.“ setzen."

Obwohl die Türkei den EUBeitritt seit Jahren anstrebt, lehnt Merkel die Aufnahme ab.

\section{Analog für Frank-Walter-} Steinmeier
Obwohl die Türkei den EU-Beitritt seit Jahren anstrebt, lehnt Merkel die Aufnahme ab.

Analog für Frank-Walter-Steinmeier 